\title{
Wireless Sensor Network for Ambient Assisted Living
}

\author{
Juan Zapata and Francisco J. Fernández-Luque and Ramón Ruiz \\ Universidad Politécnica de Cartagena \\ Spain
}

\section{Introduction}

There is a great demand, in both the public and the private sector, to take the actions needed to expand uses for electronic devices, assistive and monitoring software, and home health communication technologies to provide assisted living and health care to those in need. By means of using Information and Communication Technologies (ICT) to assist and monitor elderly, disabled, and chronically ill individuals in the home can improve quality of life, improve health outcomes, and help control health care.

Assisted living technologies are for people needing assistance with Activities of Daily Living (ADLs) but wishing to live for as long as possible independently. Assisted living exists to bridge the gap between independent living and nursing homes. Residents in assisted living centers are not able to live by themselves but do not require constant care either. Assisted living facilities offer help with ADLs such as eating, bathing, dressing, laundry, housekeeping, and assistance with medications. Many facilities also have centers for medical care; however, the care offered may not be as intensive or available to residents as the care offered at a nursing home. Assisted living is not an alternative to a nursing home, but an intermediate level of long-term care appropriate for many seniors. Increasing health care costs and an aging population are placing significant strains upon the health care system. Small pilot studies have shown that meeting seniors needs for independence and autonomy, coupled with expanded use of home health technologies, mitigate against the circumstances above, and provide improved health outcomes. Difficulty with reimbursement policies, governmental approval processes, and absence of efficient deployment strategies has hampered adopting such technologies.

Most efforts to-date of applying ICT to health care in the home have focused on telemedicine, in which ICT is used to connect the patient and his biometric information to a health care provider either in real-time or into storage for analysis at a later time (Biemer \& Hampe, 2005; Lubrin et al., 2006; Ross, 2004; Rowan \& Mynatt, 2005). While such functionality will be included in Information Technology for Assisted Living at Home (ITALH), the main objective in tele-assistence is to provide smart monitors and sensors that will alert the user and/or their care provider (be that a doctor, nurse, family member, neighbor, friend, etc.) of events such as accidents or acute illness and to diagnostic events that could indicate a deterioration in his health condition. This differs from efforts (Sixsmith \& Johnson, 2004a), in which IR sensors array are used to detect such events as accidental falls, in that the sensors themselves detect 
the event, not a central system. This reduces wireless bandwidth and greatly improves the privacy of the system by not streaming data constantly.

In particular, the chapter is focused in the introduction of an ubiquitous wireless network infrastructure to support an assisted living at home system, called DIA (Dispositivo Inteligente de Alerta, in spanish) which is being developed by Universidad Politécnica de Cartagena, Universidad de Murcia and Ambiental Intelligence \& Interaction S.L.L. (Ami2) company. Specifically, the system is constructed based on a wireless communication network in order to transfer data and events of elderly. A typical scenario consists of a private home which is instrumented based on WSN. In this context, the concept of a individual assisted by monitoring via radio-frequency is evident. The wireless infrastructure is a heterogeneous and ubiquitous, being present everywhere at once, wireless network that connects sensor devices within the home to a central Home Health System Gateway and/or a mobile Gateway. The sensor nodes themselves have embedded processing capability and are required to transmit only occasional information about their own status and messages notifying the central system when they detect a significant event. The central system, in a smart sense, connect this network to the outside world via secure Internet and telephone service so that intelligent alerts can be sent out, and authorized caregivers can have access to the system to check up on the user. Privacy and security are fundamental concerns in these systems. The chapter is organized as follows. Section 2 provides an overview of the state of the art in terms of sensor network technology. Section 3 explains the application scenario and where and how the node sensors was deployed and Section 4 discusses data processing issues. The chapter concludes with a brief summary and some final remarks.

\section{Technology Overview}

\subsection{Sensor Network Technology Overview}

A sensor network is an infrastructure comprised of sensing (measuring), computing, and communication elements that gives an administrator the ability to instrument, observe, and react to events and phenomena in a specified environment (Sohraby et al., 2007). Typical applications include, but are not limited to, data collection, monitoring, surveillance, and medical telemetry. In addition to sensing, one is often also interested in control and activation.

There are four basic components in a sensor network: (1) a set of distributed or localized sensors; (2) a communication network (usually, but not always, wireless-based); (3) a central point of information clustering (usually called base station or sink); and (4) a set of computing resources at the central point (or beyond, e.g., personal computer board or other device like PDA) to handle data correlation, event trending, status querying, and data mining. In this context, the sensing and computation nodes are considered part of the sensor network; in fact, some of the basic computation may be done in the network itself. The computation and communication infrastructure associated with sensor networks is often specific to this environment and rooted in the device and application-based nature of these networks. Figure 1 shows a generic protocol stack model that can be utilized to describe the WSN. Issues here relate to the following: (1) Physical layer treats about connectivity and coverage. (2) Data Link Layer is the protocol layer which transfers data between adjacent network nodes in a wide area network or between nodes on the same local area network segment. (3) Network Layer is responsible for end-to-end (source to destination) packet delivery including routing through intermediate hosts, whereas the data link layer is responsible for node-to-node (hop-to-hop) frame delivery on the same link. (4) Transport Layer is a group of methods and protocols 
within a layered architecture of network components within which it is responsible for encapsulating application data blocks into data units. (5) Upper (application, presentation, session) layer treats about processing application, in what could be an environment with highly correlated and time-dependent arrivals.

Management planes are needed, so that sensor nodes can work together in a power efficient way, route data in a wireless (mobile or not) sensor network, and share resources between sensor nodes. Without them, each sensor node will just work individually. From the whole sensor network point of view, it is more efficient if sensor nodes can collaborate with each other, so the lifetime of the sensor networks can be prolonged (Kahn et al., 1999).

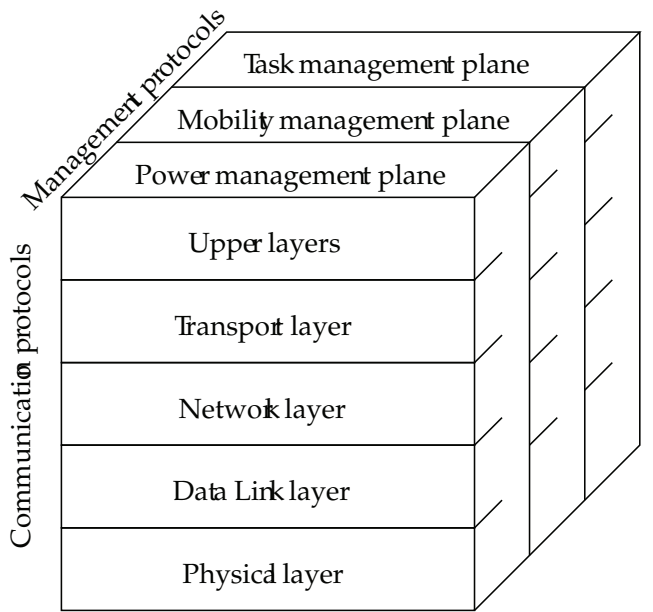

Fig. 1. Generic protocol stack for sensor networks

Sensors in a WSN have a variety of purposes, functions, and capabilities. Sensor networking is a multidisciplinary area that involves, among others, radio and networking, signal processing, artificial intelligence, database management, systems architectures for operator-friendly infrastructure administration, resource optimization, power management algorithms, and platform technology (hardware and software, such as operating systems)

The technology for sensing and control includes electric and magnetic field sensors; radiowave frequency sensors; optical-, electrooptic-, and infrared sensors; radars; lasers; location/navigation sensors; seismic and pressure-wave sensors; environmental parameter sensors (e.g., wind, humidity, heat); and biochemical national security oriented sensors. Todays sensors can be described as smart inexpensive devices equipped with multiple onboard sensing elements; they are low-cost low-power untethered multifunctional nodes that are logically homed to a central sink node. Sensor devices, or wireless nodes (WNs), are also (sometimes) called motes. Therefore, a WSN consists of densely distributed nodes that support sensing, signal processing, embedded computing, and connectivity; sensors are logically linked by selforganizing means. WNs typically transmit information to collecting (monitoring) stations that aggregate some or all of the information. WSNs have unique characteristics, such as, but not limited to, power constraints and limited battery life for the WNs, redundant data acquisition, low duty cycle, and, many-to-one flows. Power efficiency in WSNs is generally accomplished in three way: Low-duty-cycle operation, Local/in-network processing to reduce data volume 
(and hence transmission time), and multihop. Multihop networking reduces the requirement for long-range transmission since signal path loss is an inverse exponent with range or distance. Each node in the sensor network can act as a repeater, thereby reducing the link range coverage required and, in turn, the transmission power.

For a number of years, vendors have made use of proprietary technology for collecting performance data from devices. In the early 2000s, sensor device suppliers were researching ways of introducing standardization, first designers ruled out Wi-Fi (wireless fidelity, IEEE 802.11b) standards for sensors as being too complex and supporting more bandwidth than is actually needed for typical sensors. Infrared systems require line of sight, which is not always achievable; Bluetooth (IEEE 802.15.1) technology was at first considered a possibility, but it was soon deemed too complex and expensive. This opened the door for a new standard IEEE 802.15.4 along with ZigBee (more specifically, ZigBee comprises the software layers above the newly adopted IEEE 802.15.4 standard and supports a plethora of applications). IEEE 802.15.4 operates in the $2.4 \mathrm{GHz}$ industrial, scientific, and medical (ISM) radio band and supports data transmission at rates up to $250 \mathrm{kbit} \mathrm{s}^{-1}$ at ranges from 10 to $70 \mathrm{~m}$. ZigBee/IEEE 802.15 .4 is designed to complement wireless technologies such as Bluetooth, $\mathrm{Wi}-\mathrm{Fi}$, and ultra-wideband (UWB).

\subsubsection{Requeriments for Wireless Sensor Network in Ambient Assisted Living Environments}

Wireless sensor network integration in Ambient Assisted Living frameworks is usually not described in the literature, and normally is treated as a black box. Following, a list of the functional requirements for WSN in Ambient Assisted Living environments which need to be addressed in order to have a reliable system based on WSN (Martin et al., 2009) is enumerated.

1. Ambient Assisted Living Systems: Type of Networks. The design objectives are related to the development of an Ambient Assisted Living System capable of offering its services at home and nursing houses. The AAL systems could be composed by:

- A Body Sensor Network (BSN), which will include all the devices that a person must wear (accelerometers, gyroscopes, spirometers, oxymeters, etc) or use to allow the services to work. Depending on the elderly profile and the services to be configured, the BSN may include continuous monitoring sensors and other health sensors. To configure the BSN, it is always important to bear in mind the usability restrictions imposed by the users acceptance of personal devices in home environments. Basically, BSN is a mobile subset of the Wireless Sensor Network.

- A Wireless Sensor Network, which will include home infrastructure sensors (ambient, presence, pressure, home automation sensors, etc.), actuators and appliances capable of notifying their status. The Wireless Sensor Network station base will be able to communicate with the BSN by using ad hoc networking capabilities. It will include local intelligent features to dispatch events and orders depending on the situation. These processing capabilities will be part of a home gateway which will connect the home ambient via station base with the Core Care Network.

- A Core Care Network, serving as a bridge of communication between the home sensorial infrastructure and third parties and service providers (caregivers). Services may be enabled through the Core Care Network. It can also authorize the connection of external service providers, centralize system monitoring and guarantee the security of personal data. 
2. Ambient Assisted Living Systems: General design requirements for WSN. The previous scenario imposes some functional requirements to the Wireless Sensor Network finally composed by infrastructure and personal sensing nodes. Next there is a brief list of the most important features to consider.

- Interoperability. Wireless Sensor Networks in real deployments need to be ready to manage heterogeneous sensors, which need to share a common communication scheme.

- Network self-configuration and maintenance. It is desirable that the WSN demands as little attention from a human operator as possible.

- Easy and robust deployment. When designing WSN functionalities, it is important to consider the deployment requirements to make the network fully operational.

- Multihop routing. A WSN for AAL usually consists of several sensor nodes that send their measurements to a sink node, which collects all the information and typically sends it to a PC, where all the data are stored and elaborated. In a home, the sink node may not provide coverage over the whole area. As a consequence, it is necessary to implement routing algorithms that transmit the information towards the sink through other nodes.

- Positioning service. This kind of service is required for many operational and service purposes. For instance, to process the information related to the place where the user is, avoiding the storage and computation of information that is not relevant in a specific moment.

- Energy saving strategies. As the devices that are integrated in the network have limited computational and radio communication capabilities, collaborative algorithms with energy-aware communication are required to achieve multi-modal collaboration and energy conservation.

- Scalability of sensors and actuators. AALs services may impose different type of sensing and actuation requirements. For example, in a scenario considering services for COPD patients, devices sensing the quality of air may be needed. For that reason, WSN for ACSs need to be ready to include new sensors and actuators, which may be connected to existent network nodes or configure as nodes themselves. Methodologies and software architectures making easier to scale the network sensing capabilities are needed.

- Security. As wireless networks are based on a standard and data are sent over a broadcast channel, it is possible to make packet sniffing and data spoofing attacks. IEEE 802.15.4 MAC layer offers some facilities which can be used by upper layers to achieve a good level of security.

\subsection{Sensor Node Technology Overview}

Figure 2 shows the general architecture soft and hardware of a sensor node. The terms sensor node, wireless node (WN), Smart Dust, mote, and COTS (commercial off-the-shelf) mote are used somewhat interchangeably in the industry; the most general terms used here are sensor node and WN. WSNs that combine physical sensing of parameters such as temperature, light, or others events with computation and networking capabilities are expected to become ubiquitous in the next future. 

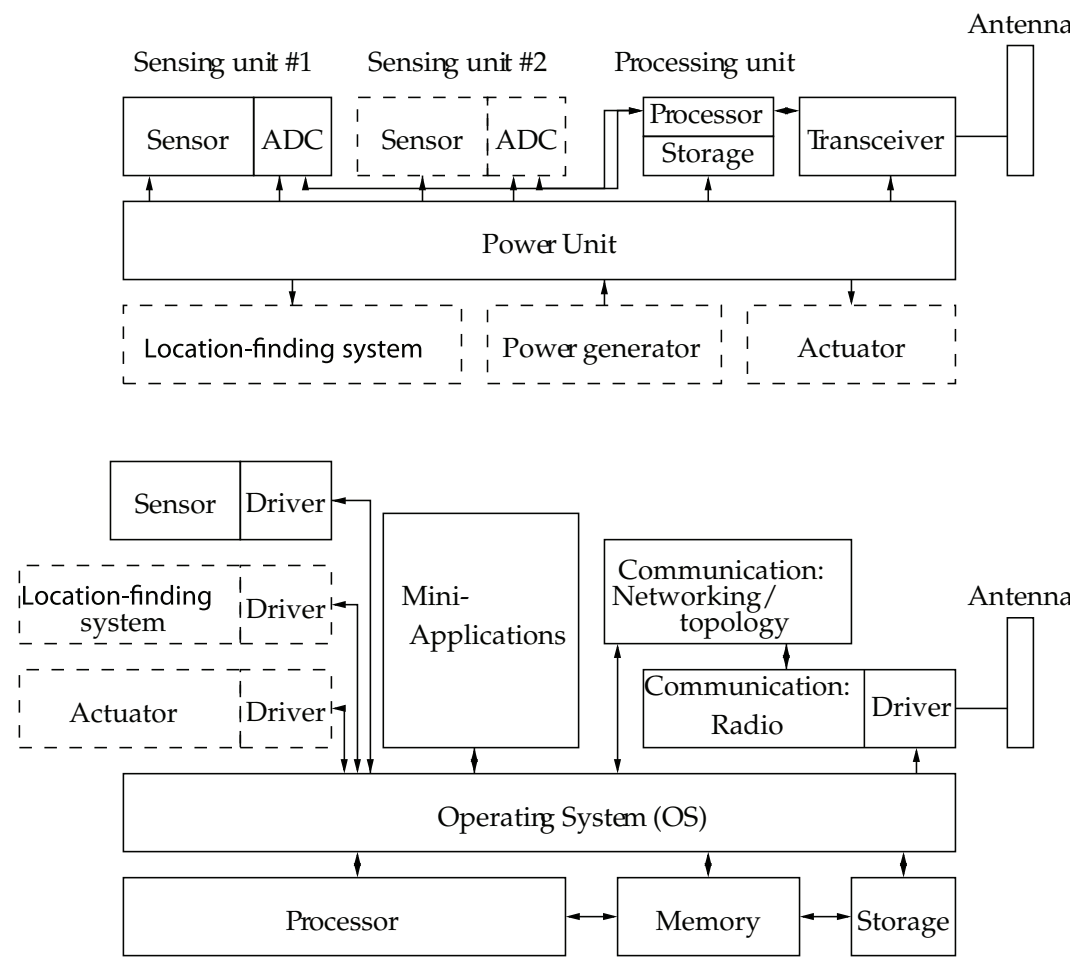

Fig. 2. Hardware and software components of WNs

Many of these examples share some basic characteristics. In most of them, there is a clear difference between sources of data the actual nodes that sense data and sinks nodes where the data should be delivered to. The interaction patterns between sources and sinks show some typical patterns. The most relevant ones are:

- Event detection. Sensor nodes should report to the sink(s) once they have detected the occurrence of a specified event. The simplest events can be detected locally by a single sensor node in isolation (e.g., a temperature threshold is exceeded); more complicated types of events require the collaboration of nearby or even remote sensors to decide whether a (composite) event has occurred (e.g., a temperature gradient becomes too steep). If several different events can occur, event classification might be an additional issue.

- Periodic measurements. Sensors can be tasked with periodically reporting measured values. Often, these reports can be triggered by a detected event; the reporting period is application dependent.

- Function approximation and edge detection. The way a physical value like temperature changes from one place to another can be regarded as a function of location. A WSN can be used to approximate this unknown function (to extract its spatial characteristics), using a limited number of samples taken at each individual sensor node. This approximate mapping should be made available at the sink. How and when to update this 
mapping depends on the applications needs, as do the approximation accuracy and the inherent trade-off against energy consumption. Similarly, a relevant problem can be to find areas or points of the same given value. An example is to find the isothermal points in a forest fire application to detect the border of the actual fire. This can be generalized to finding edges in such functions or to sending messages along the boundaries of patterns in both space and/or time.

- Tracking. The source of an event can be mobile (e.g., an intruder in surveillance scenarios). The WSN can be used to report updates on the event sources position to the $\operatorname{sink}(\mathrm{s})$, potentially with estimates about speed and direction as well. To do so, typically sensor nodes have to cooperate before updates can be reported to the sink.

Embedded sensing refers to the synergistic incorporation of sensors in structures or environments; embedded sensing enables spatially and temporally dense monitoring of the system under consideration (e.g., a home). In biological systems, the sensors themselves must not affect the system or organism adversely. The technology for sensing and control includes electric and magnetic field sensors; radio-wave frequency sensors; optical-, electrooptic-, and infrared sensors; radars; lasers; location and navigation sensors; seismic and pressure-wave sensors; environmental parameter sensors (e.g., wind, humidity, heat); and biochemical national security oriented sensors.

Small, low-cost, robust, reliable, and sensitive sensors are needed to enable the realization of practical and economical sensor networks. Although a large number measurements are of interest for WSN applications, commercially available sensors exist for many of these measurements. Sensor nodes come in a variety of hardware configurations: from nodes connected to a LAN and attached to permanent power sources, to nodes communicating via wireless multihop RF radio powered by small batteries. The trend is toward very large scale integration (VLSI), integrated optoelectronics, and nanotechnology; in particular, work is under way in earnest in the biochemical arena.

\subsubsection{Hardware and software architecture of WNs}

Normally, the hardware components of a WN include the sensing and actuation unit (single element or array), the processing unit, the communication unit, the power unit, and other application-dependent units. Sensors, particularly Smart Dust and COTS motes, have four basic hardware subsystems:

1. Sensor transducer(s). The interface between the environment and the $\mathrm{WN}$ is the sensor. Basic environmental sensors include, but are not limited to, acceleration, humidity, light, magnetic flux, temperature, pressure, and sound.

2. Computational logic and storage. These are used to handle onboard data processing and manipulation, transient and short-term storage, encryption, digital modulation, and digital transmission.

3. Communication. WNs must have the ability to communicate either in C1-WSN arrangements (mesh-based systems with multi-hop radio connectivity among or between WNs, utilizing dynamic routing in both the wireless and wireline portions of the network), and/or in C2-WSN arrangements (point-to-point or multipoint-to-point systems generally with single-hop radio connectivity to WNs, utilizing static routing over the wireless network with only one route from the WNs to the companion terrestrial or wireline forwarding node). 
4. Power supply. An appropriate energy infrastructure or supply is necessary to support operation from a few hours to months or years (depending on the application).

Sensors typically have five basic software subsystems:

1. Operating system (OS) microcode (also called middleware). This is the board common microcode that is used by all high-level node-resident software modules to support various functions. As is generally the case, the purpose of an operating system is to shield the software from the machine-level functionality of the microprocessor. It is desirable to have open-source operating systems designed specifically for WSNs; these OSs typically utilize an architecture that enables rapid implementation while minimizing code size. TinyOS is one such example of a commonly used OS.

2. Sensor drivers. These are the software modules that manage basic functions of the sensor transceivers; sensors may possibly be of the modular/plug-in type, and depending on the type and sophistication, the appropriate configuration and settings must be uploaded into the sensor (drivers shield the application software from the machine-level functionality of the sensor or other peripheral).

3. Communication processors. This code manages the communication functions, including routing, packet buffering and forwarding, topology maintenance, medium access control (e.g., contention mechanisms, direct-sequence spread-spectrum mechanisms), encryption, and FEC, to list a few.

4. Communication drivers (encoding and the physical layer). These software modules manage the minutia of the radio channel transmission link, including clocking and synchronization, signal encoding, bit recovery, bit counting, signal levels, and modulation.

5. Data processing mini-apps. These are numerical, data-processing, signal value storage and manipulations, or other basic applications that are supported at the node level for in-network processing.

\subsection{A Survey of Sensor Nodes for Wireless Sensor Networks}

A significant and prime research of sensor network is a project named Smart-Dust developed by University of California at Berkeley, USA (Pister, 2008) in the late 90s. The main objective of the project was to develop a compact size node that includes sensor, capability to compute the sensor data onboard, low cost, minimal power consumption and having bidirectional wireless communication capability. It was later sold commercially by Crossbow Inc. and Moteiv. The first mote was the WeC, which appeared in 1998 and was followed in the next year by the René mote and the next year by the René2 mote and Dot mote. Based on the field trials of these platforms, a second-generation platform called MICA was developed; it appeared in 2001. A third generation of motes were named MICA2 which was appeared in 2002. MICAz was appeared in 2002 and it was the first generation with a $2.4 \mathrm{GHz}$ frequency of radio. Iris Mote is built upon the IEEE 802.15.4 standard. It is regarded as the successor of MICAz, one of the most commonly used mote systems in the world. The most recent significant step in commercial mote development was the Telos in 2004 y Telosb in 2005.

Another interesting research testbed was the Spec platform (JLHLabs, 2008), which integrated the functionality of Mica onto a single $5 \mathrm{~mm}^{2}$ chip. Spec was built with a micro-radio, an analog-to-digital converter, and a temperature sensor on a single chip, which lead to a 30-fold reduction in total power consumption. This single-chip integration also opened the path to low cost sensor nodes. The integrated RAM and cache memory architecture greatly simplified the design of the mote family. However, the tiny footprint also requires a specialized operating 
system, which was developed by UC Berkeley, called TinyOS (TinyOS, 2009). TinyOS features component based architecture and event driven model that are suitable for programming with small embedded devices, such as motes. The combination of Motes and TinyOS is gradually becoming a popular experimental platform for many research efforts in the field of WSNs.

The Medusa MK-2 (UCLA, 2009) sensor node was carried out by the Center for Embedded Networked Sensing (CENS) at UCLA in 2002 to target both high and low-end processing applications. It integrates two microcontrollers, the first one; ATmega128 was dedicated to less computationally demanding tasks, including radio base band processing and sensor sampling. The second one, AT91FR4081, was a more powerful microcontroller $(40 \mathrm{MHz}, 1 \mathrm{MB}$ flash, $136 \mathrm{kB}$ RAM) that was designed to handle more sophisticated, but less frequent signal processing tasks (e.g., the Kalman filter). The combination of these two microcontrollers provided more flexibility in WSN development and deployment, especially for applications that require both high computation capabilities and long lifetime.

In 2002 the Berkeley Wireless Research Center (BWRC) developed System on Chip (SoC) based sensor node, named PicoNode II (Cho et al., 2005). It was built using two ASIC chips that implemented the entire node functionality. In the following year, the same team developed a first radio transmitter (that used power less than $400 \mu \mathrm{W}$ ), PicoBeacon was purely powered by solar and vibrational energy sources.

Another ASIC based approach was taken by the $\mu A M P S$ group from MIT. Following their first testbed, $\mu A M P S-I$ (MIT, 2008), the team then tried to build a highly integrated sensor node comprised of a digital and an analog/RF ASIC, $\mu A M P S-I I$. The interesting feature of $\mu$ AMPS-II was the nodes capability to operate in several modes. It can operate either as lowend stand-alone guarding node, a fully functional node for middle-end sensor networks or as a companion component in a more powerful high-end sensor system. Thus, it favored a network with heterogeneous sensor nodes for a more efficient utilization of resources.

The Free2move wireless sensor node (Bilstrup \& Wiberg, 2004) is based on a transceiver operating in the $2.4 \mathrm{GHz}$ ISM band. The node was initially thought of as an active RFID tag for monitoring temperature in goods. However, it has been shown that it is also possible to use it as a wireless sensor network node. The node is equipped with an extremely low power microcontroller (Microchip PIC16F87), for executing communication protocols and sensor functionality. The memory and processing resources are very limited to keep the price and energy consumption as low as possible. The node is also equipped with a temperature sensor.

\section{Application Scenario}

A first prototype scenario has been developed in which a user will have a home assistence system that is able to monitor his or her activity in order to detect incidents and uncommon activities (Fernández-Luque et al., 2009) and (Botía-Blaya et al., 2009). The prototype house or scenario has a bedroom, a hall, a corridor, a toilet, a kitchen, and a living room. Movement infrared sensors are installed in each location. Moreover, in the bedroom there is a pressure sensor in bed; in the hall, a magnetic sensor to detect the opening and closening of the entrance door, and in the sofa of living room another pressure sensor. All sensor boards have a complementary temperature sensor. The data is gathered from sensors mounted in the home. The sensor events are transmitted by the wireless sensor network to the base station by means ZigBee technology. A gateway is also included in the system to allow continuous monitoring. The gateway receives the events from the sensors through base station and decides what the appropriate action to take will be. Options could include querying the user to check on their status, storing (or forwarding) data on the event for future analysis by a assistential care 


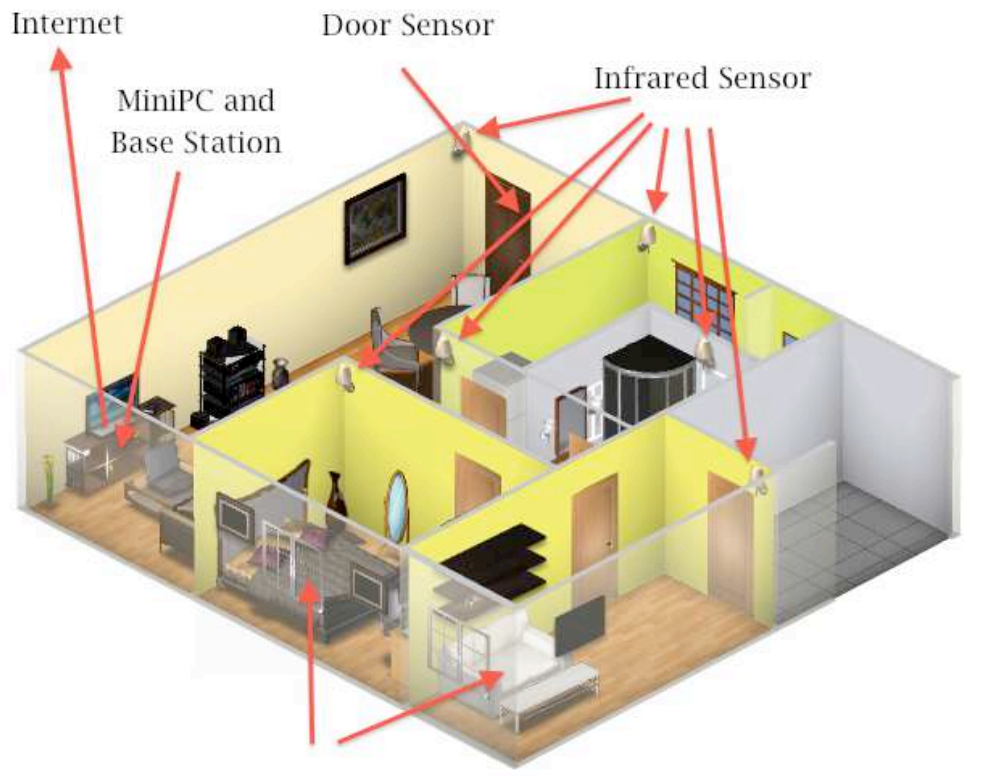

Pression

Sensor

Fig. 3. Schematic overview of the system installed at prototype home

provider, placing a telephone call to a care provider, relative or health care service, or other options. Figure 3 shows a schematic overview of the system.

The main idea consists in monitoring the person living alone in his home without interacting with him. To start, it is needed to know if he is at home in order to activate the ubiquitous custodial care system. It is easy to know by the context if a resident is at home knowing that the entrance door was opened and movement in the hall was detected. By means of distributed sensors installed in each room at home we can know the activities and the elderly location. The sensor boards developed by us allow to distinguish if movement event is induced by a pet or person thanks to a dual passive infrared sensor. On the other hand, as the pressure sensors are located in the bed and the favorite sofa in the living room, we can know more of where he is even if he is not in movement. All this sensorial assembly will be ruled by an artificial intelligent software which will allow to learn of elderly diary activities. If the system detects a suspicious event, i.e., movement in any room at 12 a.m and pressure in the bed, then the system give an alert to the caregiver.

\subsection{Assembly of Distributed Sensors}

The terms sensor node, wireless node (WN), Smart Dust, mote, and COTS (commercial offthe-shelf) mote are used somewhat interchangeably in the industry; the most general terms used here are sensor node and WN. WSNs that combine physical sensing of parameters such 
$M O \mathbb{E} B O A R D$

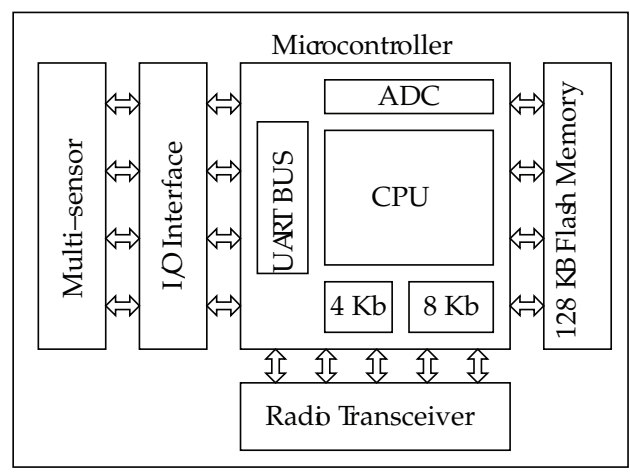

Fig. 4. Sensor node scheme

as temperature, light, or others events with computation and networking capabilities are expected to become ubiquitous in the next future. The basic functionality of a WN generally depends on the application and type of sensor device. Sensors are either passive or active devices. Passive sensors in single-element form include, among others, seismic-, acoustic-, strain-, humidity-, and temperature-measuring devices. Passive sensors in array form include optical- (visible, infrared $1 \mathrm{~mm}$, infrared $10 \mathrm{~mm}$ ) and biochemical-measuring devices. Arrays are geometrically regular clusters of WNs (i.e., following some topographical grid arrangement). Passive sensors tend to be low-energy devices. Active sensors include radar and sonar; these tend to be high-energy systems.

Activity monitoring can be beneficial for elderly people who live alone at home. By means of using electronic technologies to assist and monitor elderly, disabled, and chronically ill individuals in the home can improve quality of life, improve health outcomes, and help control asistential care. This is done with mote devices developed ad hoc for this purpose which are based on Iris mote from Crossbow (Horton \& Suh, 2005). The mote board developed uses a single channel $2.4 \mathrm{GHz}$ radio to provide bi-directional communications at $40 \mathrm{kbit} \mathrm{s}^{-1}$, and an Atmel Atmega 1281 microcontroller running at $8 \mathrm{MHz}$ controls the signal sampling and data transmission. The wireless sensor node is powered by a pair conventional AA batteries and a DC boost converter provides a stable voltage source. Figure 4 and 5 shows a schematic overview of sensor node architecture and station base architecture.

This mote board was designed in order to provide basic environmental sensing, and expansion for other sensing functionality. Actually, wearable sensors are also being included which could measure and analyze the users health as biomedicals signals (ECG, heart rate, etc) and activity such falls. Among other things because we have implemented an integrated antenna on the same board. The assembly of distribuited sensors are integrated in a mesh network. A mesh network is a generic name for a class of networked embedded systems that share several characteristics including: Multi-Hop- the capability of sending messages peer-to-peer to a base station, thereby enabling scalable range extension; Self-Configuring- capable of network formation without human intervention; Self-Healing-capable of adding and removing network nodes automatically without having to reset the network; and Dynamic Routingcapable of adaptively determining the route based on dynamic network conditions (i.e., link quality, hop-count, gradient, or other metric). Multihop protocol is a full featured multi-hop, 
- The Motes: ProDIA Sensor v2.00 (PDS200)
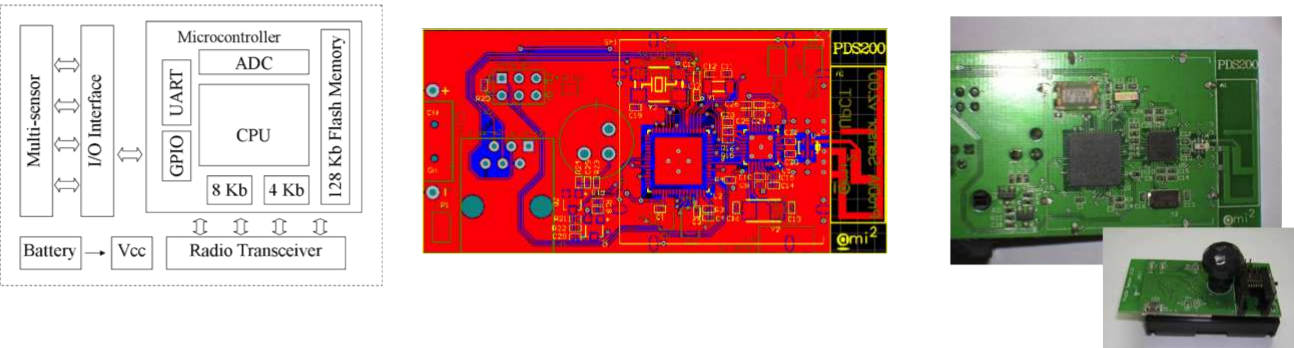

- The Base Station: ProDIA Sensor Base v1.00 (PDSB1UU)
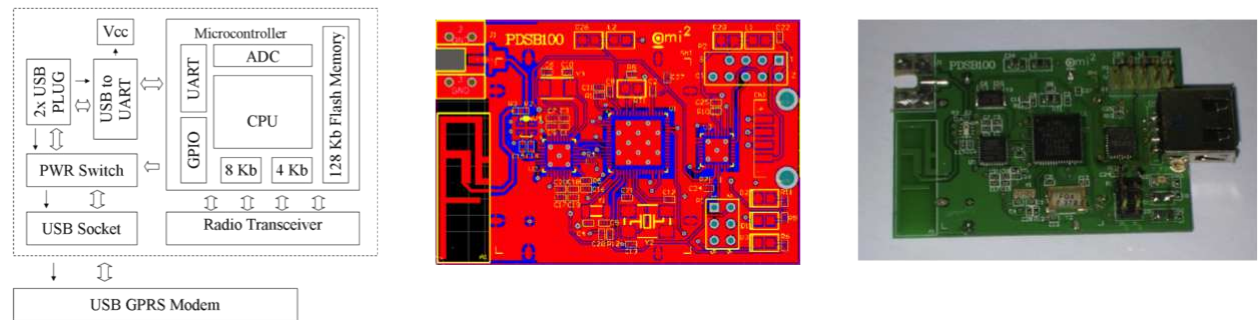

Fig. 5. Motes for PRO(totype)DIA project

ad-hoc, mesh networking protocol driven for events (Al-Karaki \& Kamal, 2004; Li et al., 2008; Sagduyu \& Ephremides, 2004). This protocol is a modified protocol based on Xmesh developed by Crossbow for wireless networks. A multihop network protocol consists of WN (Motes) that wirelessly communicate to each other and are capable of hopping radio messages to a base station where they are passed to a PC or other client. The hopping effectively extends radio communication range and reduces the power required to transmit messages. By hopping data in this way, our multihop protocol can provide two critical benefits: improved radio coverage and improved reliability. Two nodes do not need to be within direct radio range of each other to communicate. A message can be delivered to one or more nodes inbetween which will route the data. Likewise, if there is a bad radio link between two nodes, that obstacle can be overcome by rerouting around the area of bad service. Typically the nodes run in a low power mode, spending most of their time in a sleep state, in order to achieve multi-year battery life. On the other hand, the node is woke up when a event happened by means of an interruption which is activated by sensor board when an event is detected. Also, the mesh network protocol provides a networking service that is both self-organizing and selfhealing. It can route data from nodes to a base station (upstream) or downstream to individual nodes. It can also broadcast within a single area of coverage or arbitrarily between any two nodes in a cluster. QOS (Quality of Service) is provided by either a best effort (link level acknowledgement) and guaranteed delivery (end-to-end acknowledgement). Also, XMesh can be configured into various power modes including HP (high power), LP (low power), and ELP (extended low power). 
(C) 2010 The Author(s). Licensee IntechOpen. This chapter is distributed under the terms of the Creative Commons Attribution-NonCommercialShareAlike-3.0 License, which permits use, distribution and reproduction for non-commercial purposes, provided the original is properly cited and derivative works building on this content are distributed under the same license. 